\title{
Enhanced performance low-noise Brillouin ring laser for Brillouin sensing
}

Diego Marini, Leonardo Rossi, Filippo Bastianini, Gabriele Bolognini

Diego Marini, Leonardo Rossi, Filippo Bastianini, Gabriele Bolognini, "Enhanced performance low-noise Brillouin ring laser for Brillouin sensing," Proc. SPIE 11199, Seventh European Workshop on Optical Fibre Sensors, 111993I (16 September 2019); doi: 10.1117/12.2539987

Event: Seventh European Workshop on Optical Fibre Sensors, 2019, Limassol, Cyprus 


\title{
Enhanced low-noise Brillouin ring laser for Brillouin sensing
}

\author{
Diego Marini ${ }^{\mathrm{a}}$, Leonardo Rossi ${ }^{\mathrm{a}, \mathrm{b}}$, Filippo Bastianini ${ }^{\mathrm{c}}$, Gabriele Bolognini ${ }^{\mathrm{a}}$

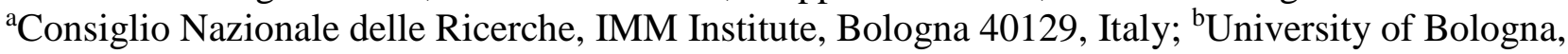 \\ Bologna 40126,Italy; 'Sestosensor s.r.l., Bologna 40069, Italy
}

\begin{abstract}
In this work, we present an enhanced design for a Brillouin Ring Laser (BRL) based on a doubly-resonant cavity (DRC) with short fiber length, paired with a heterodyne-based wavelength-locking system, to be employed as pump-probe source in Brillouin sensing applications. The enhanced source is compared with the long-cavity (LC) $(\sim 2 \mathrm{~km})$ BRL pump-probe source that we have recently demonstrated, showing a significantly lower relative intensity noise $(\sim-145 \mathrm{~dB} / \mathrm{Hz}$ in the whole $0-800 \mathrm{MHz}$ range), a narrower linewidth $(10 \mathrm{kHz})$, combined with large tunability features and an excellent pumpprobe frequency stability $(\sim 200 \mathrm{~Hz})$ which is uncommon for fiber lasers. The measurement of intensity noise on the novel BRL signal yielded an improved signal-to-noise ratio (SNR) of about $22 \mathrm{~dB}$ with respect to LC-BRL schemes that is expected to lead to a temperature/strain resolution enhancement in BOTDA applications up to $5.5 \mathrm{~dB}$.
\end{abstract}

Keywords: Distributed fiber optics sensing (DFOS), Brillouin fiber lasers (BFL), Brillouin optical time domain analysis (BOTDA).

\section{INTRODUCTION}

Brillouin Optical Time Domain Analysis (BOTDA) sensors use Stimulated Brillouin Scattering (SBS) to accurately reconstruct temperature and strain distribution along a sensing fiber achieving more than $100 \mathrm{~km}$ sensing range and submeter spatial resolution [1]. In BOTDA pump-probe schemes, a pulsed signal (pump) and a CW radiation (probe) downshifted in frequency, are launched from both ends of the sensing fiber: the probe signal is amplified through SBS with a maximum gain occurring at a pump-probe frequency difference is equal to the Brillouin frequency shift (BFS) of the sensing fiber [2]. Since the BFS is temperature and strain dependent, the measurement of the Brillouin gain spectra and in particular of the BFS values allows to reconstruct the spatial distribution of temperature and strain along the sensing fiber [3]. The techniques that are commonly employed for the generation of the pump and probe signals are based on phase-locked-loop (PLL) systems [4] and optical sideband generation (OSB) technique [5]. In [3] we have demonstrated a BOTDA systems employing a long-cavity $(\sim 2 \mathrm{~km})$ Brillouin ring laser as source for pump and probe signals achieving an accuracy in the BFS evaluation of $\sim 1 \mathrm{MHz}$ over a more than $10 \mathrm{~km}$ sensing distance. Using the BRL as BOTDA source the shift between the pump and the Stokes signal is already in the range of the Brillouin frequency shift and it is possible to tune their frequency shift using an electro-optic modulator (EOM) with a much narrower bandwidth than modulators used in the OSB method. In this work, we present an enhanced design for a Brillouin Ring Laser (BRL) to be employed in Brillouin sensing systems. The novel BRL is based on a doubly-resonant cavity (DRC) with short fiber length $(<5 \mathrm{~m})$ that allows to reduce phase and intensity noise with respect to the long-cavity version and enhance the laser conversion efficiency, paired with a heterodyne-based wavelength-locking system used to tune the pump-probe frequency shift.

\section{WAVELEGTH LOCKED SHORT-CAVITY DOUBLY RESONANT BRL}

The scheme of the proposed BRL is reported in Fig. 1(a). The core of the source is the short ring resonator made up by a $<5 \mathrm{~m}$ long single mode fiber (SMF) section closed by a directional coupler with coupling ratio 90/10 (C1). A distributed feedback (DFB) laser, with spectral linewidth $350 \mathrm{kHz}$, is used to generate the seed pump radiation oscillating at $\lambda=1.55$ $\mu \mathrm{m}$ that is injected into the loop through the circulator OC and the coupler $\mathrm{C} 1$. The cavity eigenstate of polarization (ESOP) is selected through a polarization controller inside the loop (PC1). Another polarization controller (PC2) is placed before the coupler $\mathrm{C} 1$ and is used to align the SOP of the injected radiation to the cavity ESOP in order to maximize the circulating power. The Stokes signal, circulating in the opposite direction of the pump (anti-clockwise direction in the figure), is extracted through $\mathrm{C} 1$ and $\mathrm{OC}$. The short cavity (SC) layout limits the resonant mode instability of pump and Brillouin Stokes signals induced by thermal fluctuations and acoustic noise. In particular, the ring resonator length is reduced enough so that the frequency spacing of longitudinal modes, i.e. the free spectral range $F S R$ given by $c / n_{g} L$, with c the speed of

Seventh European Workshop on Optical Fibre Sensors, edited by Kyriacos Kalli, Gilberto Brambilla, Sinead O'Keeffe Proc. of SPIE Vol. 11199, 111993I - C 2019 SPIE · CCC code: 0277-786X/19/\$21 · doi: 10.1117/12.2539987 
light, $n_{g}$ the fiber group refractive index and $\mathrm{L}$ the fiber length, becomes larger than the Brillouin gain bandwidth, making multimode lasing and mode hopping more difficult. A resonant configuration is adopted for both seed and Stokes signals in order to maximize the optical intensity of the circulating signals, increase the interaction length reducing the lasing threshold power. Doubly resonant condition is achieved when the Brillouin shift $\Delta v_{S B S}$ coincides with an integer number $m$ of the FSR:

$$
\Delta v_{S B S}=m \cdot F S R
$$

The ring cavity length has been precisely set to achieve the double resonance condition using the single-cut technique described in [6]. The ring cavity, after the removal of the excess length, was $3.4 \mathrm{~m}$ long.

A wavelength-locking scheme is used to finely tune the BRL signal frequency setting the pump-probe frequency shift to be used in BOTDA measurement. The scheme that implements the wavelength locking scheme is reported in Fig 1(b). A small fraction of both DFB pump and BRL Stokes lightwaves is extracted by two optical couplers (S1 and S2) from the BRL scheme and combined trough the 3-dB optical coupler S3. The beating signal is converted by a fast photo-detector (PD) into a RF signal oscillating at frequency $\Delta \mathrm{f}_{\mathrm{BRL}}=\mathrm{f}_{\text {pump }}-\mathrm{f}_{\mathrm{BRL}}$ and a harmonic mixer is used to mix it to the tunable local oscillator signal at the frequency $f_{L O} . f_{L O}$ is equal to the pump-probe frequency shift value that we want to set and is always kept higher than $\Delta \mathrm{f}_{\mathrm{BRL}}\left(\mathrm{f}_{\mathrm{LO}}>\Delta \mathrm{f}_{\mathrm{BRL}}\right.$, in our experiment $\left.\mathrm{f}_{\mathrm{LO}}=10.7-11.5 \mathrm{GHz}\right)$. The harmonic mixing generates two sidebands $\left(\mathrm{f}_{\mathrm{LO}} \pm \Delta \mathrm{f}_{\mathrm{BRL}}\right)$; a low-pass filter removes the (unwanted) upper sideband, while the frequency-difference $\mathrm{RF}$ component $\left(\mathrm{f}_{\mathrm{LO}}\right.$ $\Delta \mathrm{f}_{\mathrm{BRL}}$ ), which in our experiment lies in the $100-900 \mathrm{MHz}$ range, is used to drive a low-bandwidth ( $\left.<1 \mathrm{GHz}\right) \mathrm{Mach}$-Zehnder electro-optical modulator (EOM). Corresponding optical sidebands are thus generated in modulation of Stokes light by EOM, while a suitable biasing of the EOM ensures efficient suppression of the
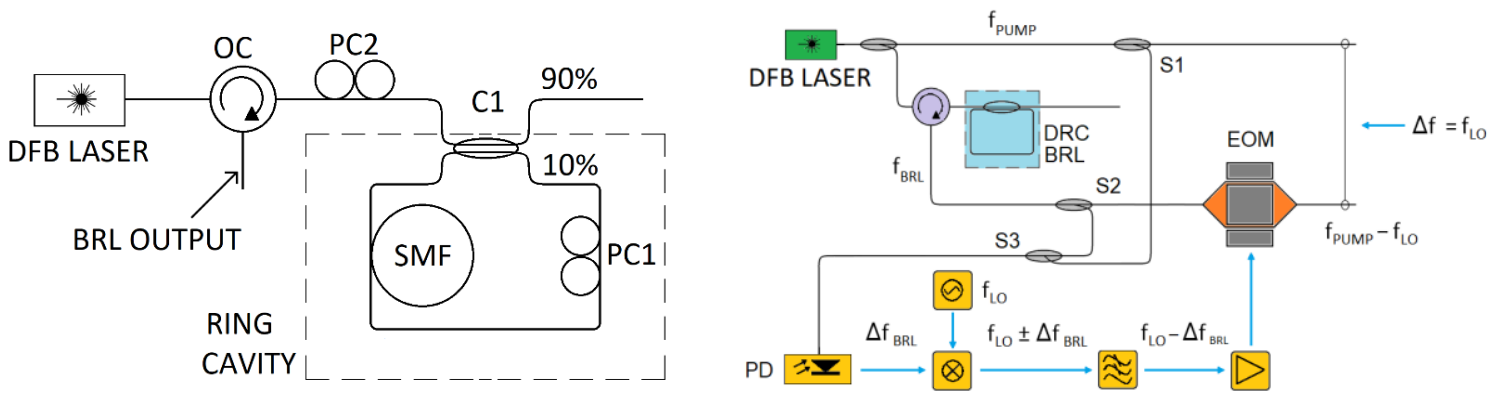

Fig. 1. Double-resonance short-cavity BRL (a) and scheme of implemented active wavelength locking technique for DRC-BRL (b).

(Stokes) carrier ( $>20 \mathrm{~dB}$ suppression). The lower sideband $\mathrm{f}_{\mathrm{LSB}}$ frequency is shifted from that of the pump by the value $f_{L O}\left(f_{L S B}=f_{B R L}+\Delta f_{B R L}-f_{L O}=f_{\text {pump }}-f_{L O}\right)$. This way, the pump-probe frequency shift is set to the local oscillator frequency $f_{L O}$ and can be easily tuned to the values required to span the whole BGS of the sensing fiber. The BRL signal frequency shift is $<10 \mathrm{GHz}$ and does not lie within the gain spectrum of the sensing fibers that are commonly employed, thus the upper sideband signal that is generated by the EOM does not interact with the pulsed pump in the BOTDA set-up and does not have to be suppressed. In addition, the wavelength locking system compensates any perturbation of BRL ring frequency $\delta \mathrm{f}$. In particular, any perturbation $\delta \mathrm{f}$ would lower the $\Delta \mathrm{f}_{\mathrm{BRL}}$ by $-\delta \mathrm{f}$, causing the frequency that is fed into the EOM to change from $\left(f_{\mathrm{LO}}-\Delta \mathrm{f}_{\mathrm{BRL}}\right)$ to $\mathrm{f}_{\mathrm{LO}}-\left(\Delta \mathrm{f}_{\mathrm{BRL}}-\delta \mathrm{f}\right)$. This would result in a modulation change of exactly $\delta \mathrm{f}$ and in a perturbed lower sideband frequency equal to $f_{L S B}=f_{B R L}+\delta f-f_{L O}+\Delta f_{B R L}-\delta f=f_{\text {pump }}-f_{L O}$, leaving the final pump-probe frequency shift unaltered. It's worth noting that OSB techniques require modulators having bandwidth $>10-11 \mathrm{GHz}$ to directly modulate the probe signal from the pump frequency values to those required to span the BGS of the sensing fiber. On the other hand, the method here presented only requires modulators with a bandwidth of around $1 \mathrm{GHz}$.

\section{CHARACTERIZATION OF DR-SC BRL}

The temporal stability of the pump-probe frequency shift, range of tunability, spectral linewidth and intensity noise of the DR-SC BRL have been measured in order to assess its possible use for the generation of pump-probe signals in BOTDA systems. In Fig. 2, we report the pump-probe frequency shift spectra obtained converting the beating signal of the two lightwave signals into electrical domain through a wide bandwidth photodetector and analyzing it at the electrical spectrum analyzer (ESA). The beating signal has been acquired for a local oscillator frequency $\mathrm{f}_{\mathrm{LO}}=10.86 \mathrm{GHz}$ and for an integration 
time of 10 and $120 \mathrm{~ms}$. The measure shows that the pump-probe frequency shift can be efficiently tuned with high accuracy: a discrepancy of less than $1 \mathrm{kHz}$ is obtained between the local oscillator frequency and the measured frequency shift. More importantly, no appreciable drifts are observed for the integration times of the measurements: for the $10 \mathrm{~ms}$ timescale, the full width half maximum (FWHM) is $\sim 200 \mathrm{~Hz}$; the FWHM for longer averaging times, up to $120 \mathrm{~ms}$ results to be of the same order of magnitude $(\sim 400 \mathrm{~Hz})$. Furthermore, we obtained a tuning range $>200 \mathrm{MHz}$, that is wider than the BGS of the sensing fibers employed in BOTDA sensors, achieving comparable accuracy and temporal stability. Then, we measured the spectral bandwidth of the BRL using the delayed self-heterodyne technique obtaining a linewidth of $\sim 10 \mathrm{kHz}$ at full width half maximum. Considering that the pump linewidth is approximately $350 \mathrm{kHz}$, a considerable enhancement in spectral purity is achieved in stabilized BRL. In particular, the narrowing effect of the cavity as well as the active wavelength-locking scheme reduce the spectral linewidth by a factor of 35, considerably increasing the coherence of the signal. Finally, we have compared the intensity noise of the wavelength-locked DR-SC BRL, free-running long-cavity ( $2 \mathrm{~km}) \mathrm{BRL}$ version and DFB laser pump. The relative intensity noise (RIN) values obtained in the frequency range $(0-800$ $\mathrm{MHz})$ are reported in Fig. 3. It can be noted that in the long-cavity BRL higher RIN values are observed in the low frequency range (0-400 MHz), while the actively stabilized DRC-BRL exhibits lower RIN across the entire frequency
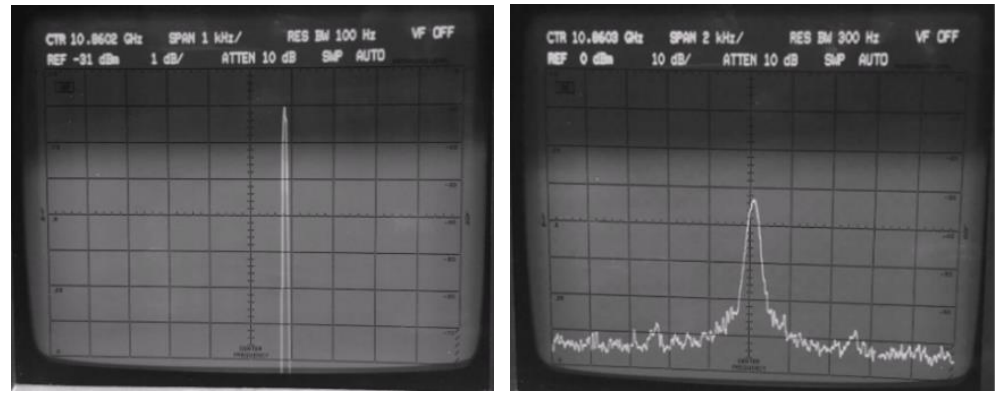

Fig. 2. Electrical spectrum of pump-probe beating for stabilized DRC-BRL with $10 \mathrm{~ms}$ (left) and $120 \mathrm{~ms}$ (right) measurement time. The frequency range is centered at the local oscillator frequencies $\mathrm{f}_{\mathrm{LO}} 10.8602 \mathrm{GHz}$ that is fed into the active wavelength-locking scheme. The $y$ axis scale is $1 \mathrm{~dB} /$ division (left ) and $10 \mathrm{~dB} / \mathrm{division}$ (right) and $\mathrm{x}$ axis scale is $1 \mathrm{kHz} /$ division (left) and $2 \mathrm{kHz} /$ division (right).

range. In particular, while for standard BRL the low-frequency maximum RIN values are about $-90 \mathrm{~dB} / \mathrm{Hz}$ (in the $10-15$ $\mathrm{MHz}$ frequency range), the RIN levels of stabilized DRC-BRL scheme are only slightly above the original pump DFB levels, i.e. between $-140 \mathrm{~dB} / \mathrm{Hz}$ and $-150 \mathrm{~dB} / \mathrm{Hz}$ across the whole $0-800 \mathrm{MHz}$ range. On the other hand, RIN values beyond $500 \mathrm{MHz}$ frequencies appear low even for the standard BRL and remain approximately constant with an average value of about $-145 \mathrm{~dB} / \mathrm{Hz}$. The RIN measurement have been used to evaluate the improvement in temperature and strain resolution of BOTDA sensor stemming from the employment of the wavelength-locked DR-SC BRL with respect to the LC-BRL scheme.

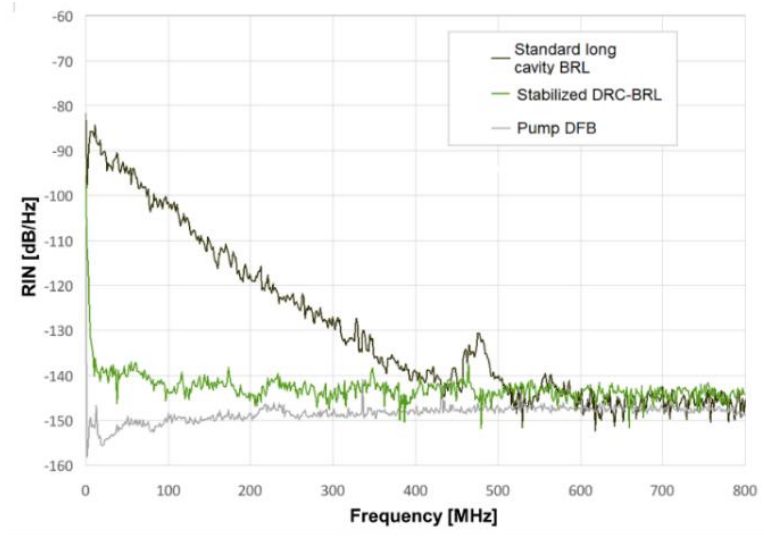

Fig. 3. Measured spectral RIN characteristics for the DR-SC BRL source, LC-BRL and DFB pump.

The main performance limit for the sensors is the signal-to-noise ratio (SNR) level of the output of the photodetector detecting the SBS-amplified probe signal. For schemes employing sources affected by intensity noise, as in the case of 
fiber lasers, RIN represents the biggest contribution to SNR degradation and ultimately limits the strain and temperature resolution [7]. In [3] we reported the results of BOTDA measurements using a long-cavity BRL for the generation of both pump and probe signals. The LC BRL showed relatively high intensity fluctuation due to mode hopping with RIN values up to $-85 \mathrm{~dB} / \mathrm{Hz}$ in sub-MHz range in line with those reported in figure for LC-BRL. Taking into consideration the RIN measurement that we have reported, the employment of the low-RIN enhanced BRL that we have demonstrated is therefore expected to significantly improve the strain and temperature resolution of the sensor. In BOTDA distributed sensor schemes, the reconstruction of the temperature and strain spatial distribution is accomplished by extracting the Brillouin frequency shift through a Lorentzian fitting of the Brillouin gain spectra along the sensing fiber. The minimum detectable change of Brillouin shift $\delta v_{B}$ can be estimated through the Brillouin linewidth of the sensing fiber and the signal SNR [7]:

$$
\delta v_{B}=\frac{\Delta v_{B}}{\sqrt{2}(S N R)^{1 / 4}}
$$

where $\Delta v_{B}$ is the FWHM of the Brillouin gain trace. Eq. (2) can be used to evaluate the BOTDA strain and temperature resolution, $\delta \varepsilon$ and $\delta T$, respectively [7]:

$$
\delta \varepsilon=\frac{\delta v_{B}}{C_{S} v_{B}(0)} \quad \delta T=\frac{\delta v_{T}}{C_{T} v_{B}\left(t_{r}\right)}
$$

where $C_{S}$ and $C_{T}$ are the linear temperature and strain coefficients, respectively. $v_{B}(0)$ and $v_{B}\left(t_{r}\right)$ represent the Brillouin frequency shifts of unstrained sensing fiber and of sensing fiber at reference temperature. The SNR values of the DR-SC BRL and LC BRL have been obtained integrating the RIN values that we have measured over the receiver bandwidth used in [3] (125 MHz), and Eqs. (2-3) are used for the evaluation of the strain-temperature resolution. From calculations, we obtained SNR values of $38 \mathrm{~dB}$ for the long cavity BRL and of $61 \mathrm{~dB}$ for the locked DR-SC BRL configuration with SNR improvement $>22 \mathrm{~dB}$. From these values it results that, in the assumption of RIN as the prevalent component in probe fluctuations and in detected SNR, the resolution improvement (in terms of frequency, temperature or stain resolution) achievable with the DRC-BRL reaches values up to $5.5 \mathrm{~dB}$.

In conclusion, we have showcased a dramatic reduction of RIN values of locked DR-SC actively stabilized BRL with respect to LC-BRL, which is predicted to provide a significant performance enhancement of the BOTDA sensor employing it as laser source. In particular, the SNR improvement of more than $22 \mathrm{~dB}$ with respect to the LC layout used for the preliminary experiments can enhance the sensitivity of temperature and strain measurement by a factor of $5.5 \mathrm{~dB}$.

\section{ACKNOWLEDGMENT}

This activity has received funding from the European Union through Horizon 2020, the Framework Programme for Research and Innovation, under project PULSe, Grant Agreement No. 737801

\section{REFERENCES}

[1] Hartog, A. H., [An introduction to distributed optical fibre sensors], CRC press, (2017).

[2] Denisov, A., Soto, M. A. and Luc Thévenaz., "Towards 1'000'000 resolved points in a distributed optical fibre sensor," Proc. SPIE 9157, 9157D2 (2014).

[3] Marini, D., Iuliano, M., Bastianini, F and Bolognini, G., "BOTDA sensing employing a modified Brillouin fiber laser probe source," J. Lightwave Technol. 36(4), 1131-1137, (2018).

[4] Nikles, M., Théveneaz, L. and Robert, P. A., "Simple distributed fiber sensor based on Brillouin gain spectrum analysis," Opt. Lett. 21(10), 758-760 (1996).

[5] Kurashima, T., Horiguchi, T. and Tateda, M., "Distributed-temperature sensing using stimulated Brillouin scattering in optical silica fibers," Opt. Lett. 15(18), 1038-1040 (1990).

[6] Spirin, V. V., et al. "Single cut technique for adjustment of doubly resonant Brillouin laser cavities," Opt. Lett. 38(14), 2528-2530 (2013).

[7] Horiguchi, T., Shimizu, K., Kurashima, T., Tateda, T. and Koyamada, Y., "Development of a distributed sensing technique using Brillouin scattering," J. Light. Technol. 13(7), 1296-1302 (1995).Hartog, A. H., [An introduction to distributed optical fibre sensors], CRC press, (2017). 